\title{
Towards Antichain Algebra
}

\author{
Bernhard Möller \\ Institut für Informatik, Universität Augsburg, D-86135 Augsburg, Germany \\ bernhard.moeller@informatik. uni-augsburg. de
}

\begin{abstract}
We use an algebra of preference strict-orders to give a formal derivation of the standard Block-Nested Loop (BNL) algorithm for computing the best or maximal objects w.r.t. such an order. This derivation is presented in terms of antichains, i.e., sets of mutually incomparable objects. We define an approximation relation between antichains that reflects the steps taken by the BNL algorithm. This induces a semilattice and the operator computing the maximal objects of a subset can be viewed as a closure operator in an associated pre-ordered set and hence yields a characterisation of antichains in terms of a Galois connection.
\end{abstract}

Keywords: preference relations, maximal objects, block-nested loop algorithm, lattice theory, Galois connections

\section{Introduction}

The motivation for this work arose in the area of preference databases (see [7). Classical databases had supported only queries with so-called hard constraints, by which the objects sought in the database are clearly and sharply characterised. Hence, if there are no exact matches the empty result set is returned, which is often very frustrating for users. As a remedy, over the last decades queries with soft constraints have been studied. These constraints arise from a formalisation of the user's preferences in the form of partial strict-orders.

For instance, a person wanting to have a vacation may prefer inexpensive hotels closer to the beach over expensive ones further off. This could be formalised as the following preference relation $\prec$ between tuples $s, t$ :

$$
\begin{aligned}
& s \prec t \Leftrightarrow_{d f} \quad(\text { t.prize }<\text { s.prize }\wedge t \text {.dist } \leq \text { s.dist }) \vee \\
&(\text { t.prize } \leq \text { s.prize } \wedge \text { t.dist }<\text { s.dist })
\end{aligned}
$$

A query with such a preference order may then return the set of "best" or maximal objects found in the search space. As usual in partial orders, the maximal objects are pairwise incomparable, i.e., form an antichain.

If the search space has two dimensions, like in the above example, it can be depicted in a $2 \mathrm{D}$ rectangular coordinate system. The maxima then are the end points of a stair-case like shape, a.k.a. the "skyline" [1, see Fig. 1. 


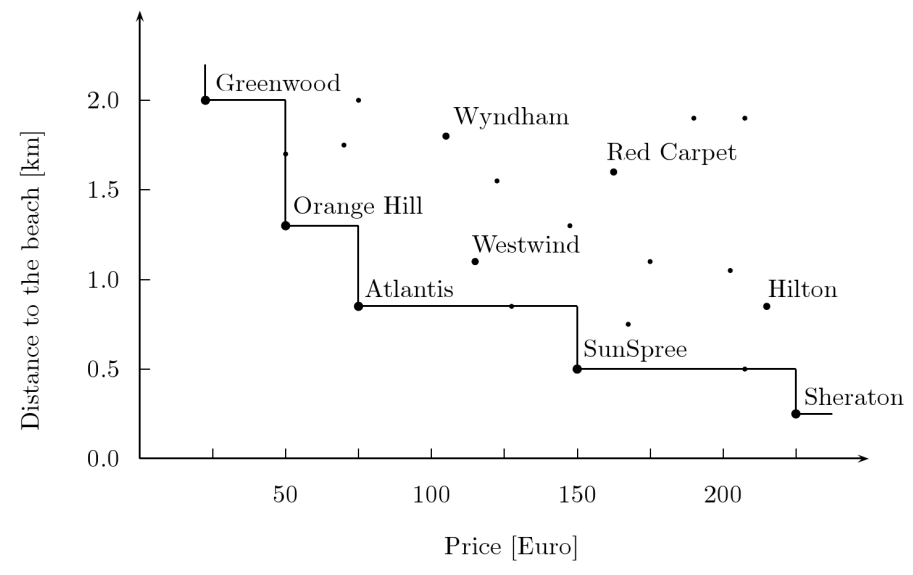

Fig. 1. A skyline diagram

In earlier papers [8, 7] we have developed an algebraic calculus for reasoning about the set $a \triangleright p$ of maximal objects in a set $p$ w.r.t. a preference relation $a$, independent of the special application area of databases. In the present paper we extend these results by a number of additional ones. As a test case we give a derivation of the standard Block-Nested Loop (BNL) algorithm (e.g. 1]) for computing the maximal objects. To the best of our knowledge this is the first calculational treatment of that algorithm. A closer analysis exhibits that there is an approximation order between antichains underlying that algorithm which even induces a semilattice structure. While, w.r.t. the inclusion order, the maxima operator $a \triangleright p$ is antitone (i.e., monotonically decreasing) in $a$, it is neither isotone (i.e., monotonically increasing) nor antitone in $p$. Fortunately, isotony can be recovered by passing to the approximation order. Last, the maxima operator can be viewed as a closure operator in an associated preordered set and hence yields a characterisation of antichains in terms of a Galois connection.

The paper is structured as follows. In Sect. 2 we recapitulate basic notions about preorders and orders as well as the algebraic notions in terms of semirings that underlie our calculus. Sect. 3 presents basic results about the algebraic representation of the maxima operator. Next to new properties concerning the relation between what we call normality of a strict-order and its noetherity, we show a couple of auxiliary results for the following sections. Sect. 4 provides various characterisations of antichains and properties concerning the maxima of a union of sets. In Sect. 5 we give the announced calculational derivation of the BNL algorithm. Sect. 6 presents an approximation order between antichains and shows that it induces a semilattice as well as some results on isotony and suprema preservation of the maxima operator. The BNL algorithm is shown to construct an ascending chain of antichains w.r.t. that order. In Sect. 7 we then prove that a modified version of the approximation order exhibits the maxima operator as a closure operator in a preordered set and hence as an adjoint in a Galois connection. Since both closures and Galois connections are usually only 
dealt with in partial orders, we provide the necessary results on the preorder case, partially in an Appendix. The paper finishes with a brief conclusion and outlook in Sect. 8 .

\section{Preliminaries}

\subsection{Preorders and Partial Orders}

A preorder is a pair $(A, \leq)$, where $A$ is a set and $\leq$ is a reflexive and transitive binary relation on $A$. The relation $\sim$ defined by $x \sim y \Leftrightarrow_{d f} x \leq y \wedge y \leq x$ is an equivalence relation, called the equivalence induced by $\leq$. If $\leq$ is also antisymmetric then $(A, \leq)$ is called an order; in this case $\sim$ coincides with equality.

A useful tool for working with preorders are the rules of indirect inequality:

$$
x \leq y \Leftrightarrow(\forall z: z \leq x \Rightarrow z \leq y), \quad x \leq y \Leftrightarrow(\forall z: y \leq z \Rightarrow x \leq z) .
$$

The direction $(\Rightarrow)$ needs transitivity of $\leq$, whereas $(\Leftarrow)$ needs reflexivity. By combining these, we obtain the rule of indirect equivalence:

$$
x \sim y \Leftrightarrow(\forall z: z \leq x \Leftrightarrow z \leq y) \Leftrightarrow(\forall z: x \leq z \Leftrightarrow y \leq z) .
$$

\subsection{Algebraic Notions}

Throughout we assume an idempotent semiring $(S,+, 0, \cdot, 1)$. This means that + and - are associative operators on set $S$, with neutral elements 0 and 1, resp.; moreover, + is assumed to be commutative and idempotent, i.e., to satisfy $a+a=$ $a$ for all $a \in S$. Finally, · is assumed to distribute through + in both arguments and to preserve 0, i.e., $0 \cdot a=0=a \cdot 0$.

Because of the properties of + one can define a partial order $\leq$ on $S$ by $a \leq b \Leftrightarrow{ }_{d f} a+b=b$. It is called the natural order or subsumption order. It induces an upper semilattice in which + is the binary supremum operator. If that semilattice is even a complete lattice and · distributes through arbitrary suprema then $S$ is called a quantale.

A prominent example of an idempotent semiring, that is even a quantale, is provided by the set of all binary relations over a set $M$, with union as + and relational composition as $\cdot$. The roles of 0 and 1 are played by the empty relation $\emptyset$ and the identity relation $I$. The natural order coincides with relational inclusion. Partial identity relations $I_{N}=_{d f}\{(x, x) \mid x \in N\} \subseteq I$, a.k.a. coreflexives or monotypes, can be used to encode subsets $N \subseteq M$ as relations.

Inspired by that, we model preference relations between database tuples abstractly by general semiring elements $a \in S$ and sets of database tuples by tests $p \leq 1$, analogous to the above partial identity relations. Tests $p$ are required to have a complement $\neg p$ relative to 1 , uniquely characterised by the conditions $p+\neg p=1$ and $p \cdot \neg p=0=\neg p \cdot p$.

The set of all tests of $S$ is denoted by test $(S)$; it forms a Boolean algebra with + as supremum and $\cdot$ as infimum, least element 0 and greatest element 1. 
Between tests the order $\leq$ is the abstract counterpart of set inclusion. We define the difference operator for $p, q \in \operatorname{test}(S)$ as $p-q={ }_{d f} \quad p \cdot \neg q$ and assume that it associates to the left. We also note that it is right-commutative, i.e., satisfies $p-q-r=p-r-q$.

An element $p \in \operatorname{test}(S)$ is called atomic if $p \neq 0$ and $\forall q \in \operatorname{test}(S): q \leq p \Rightarrow$ $q=0 \vee q=p$. While general tests stand for sets of database tuples, atomic tests correspond to single database tuples. Because of that we will frequently use set-theoretic terminology when talking about them, such as "all objects in $p$ " and the like. Finally, we note that an atom $x$ in a Boolean algebra satisfies $x \leq p+q \Rightarrow x \leq p \vee x \leq q$. In particular,

$$
x \not \leq p \Rightarrow x \leq \neg p .
$$

Tests are used to define the central operators of a modal semiring, namely box and diamond which can be defined in a forward and backward form. In the present note we will only use the forward diamond $|a\rangle: \operatorname{test}(S) \rightarrow \operatorname{test}(S)$, which can be axiomatised by

$$
|a\rangle q \leq p \Leftrightarrow \neg p \cdot a \cdot q \leq 0, \quad|a \cdot b\rangle q=|a\rangle|b\rangle q={ }_{d f}|a\rangle(|b\rangle q) .
$$

Informally, the test $|a\rangle q$ represents all database tuples that are $a$-related to (or dominated by) some tuple in the set represented by $q$. Hence $|a\rangle$ can be viewed as an algebraic form of the inverse image operator on binary relations. In particular, the domain of element $a$ can be defined as the inverse image of the largest test 1 as $\left\ulcorner a={ }_{d f}|a\rangle 1\right.$.

A corresponding forward box operator $\mid a]$ is defined as the De Morgan dual of $|a\rangle$ by $\mid a] q={ }_{d f} \neg|a\rangle \neg q$. It is an algebraic counterpart of Dijkstra's wlp operator and can be used to define an algebraic version of Hoare triples.

Diamond and box satisfy many useful laws (e.g. [3]). The most important ones for diamond are additivity (and hence isotony) in both arguments:

$$
|a+b\rangle p=|a\rangle p+|b\rangle p, \quad|a\rangle(p+q)=|a\rangle p+|a\rangle q
$$

In fact, $|a\rangle$ preserves arbitrary suprema; if $S$ is a quantale then |\rangle preserves abitrary suprema in both arguments.

If the underlying semiring is a Kleene algebra, i.e., has an operation $*$ for finite iteration with the standard axioms (e.g. 6]), we have the unfold and induction rules for the diamond of a starred element:

$$
\begin{array}{ll}
\left|a^{*}\right\rangle p=p+|a\rangle\left|a^{*}\right\rangle p, & \text { (star-dia-unfold) } \\
p \leq q \wedge|a\rangle q \leq q \Rightarrow\left|a^{*}\right\rangle p \leq q . & \text { (star-dia-induct) }
\end{array}
$$

\section{Strict-Orders and Maxima}

Definition 3.1 An element $a$ is called $d$-transitive ("d" standing for "diamond") if all tests $p$ satisfy $|a\rangle|a\rangle p \leq|a\rangle p$. By the second diamond axiom this is equivalent to $|a \cdot a\rangle p \leq|a\rangle p$. It is, however, more liberal than stipulating $a \cdot a \leq a$; for the 
case of relations both formulations coincide. An element $a$ is called $d$-irreflexive if for all atomic tests $x$ we have $x \cdot|a\rangle x \leq 0$. A d-transitive and d-irreflexive element is called a strict-order.

Corollary 3.2 For d-transitive $a$ and test $p,|a\rangle|a+1\rangle p=|a\rangle p=|a+1\rangle|a\rangle p$.

Proof. We only show the first equation; the second one is symmetric. By distributivity of diamond, $|1\rangle$ being the identity, the assumption and the definition of $\leq$ we have $|a\rangle|a+1\rangle p=|a\rangle|a\rangle p+|a\rangle|1\rangle p=|a\rangle|a\rangle p+|a\rangle p=|a\rangle p$.

In a Kleene algebra, for any d-transitive element $a$ and test $p$,

$$
\left|a^{*}\right\rangle p=|a+1\rangle p=|a\rangle p+|1\rangle p=|a\rangle p+p .
$$

Definition 3.3 The best or maximal objects w.r.t. an element $a$ and a test $p$ are represented by the test

$$
a \triangleright p={ }_{d f} p-|a\rangle p .
$$

This can be understood as follows. The expression $|a\rangle p$, the inverse image of $p$ under preference element $a$, denotes the set of objects that are dominated by some object in $p$. Hence $p-|a\rangle p$ consists of the non-dominated and hence maximal objects in $p$.

The following lemma collects useful properties of the maximality operator; proofs can be found in 7 .

Lemma 3.4 The following holds for arbitrary elements $a, b$ and test $p$ :

1. $a \triangleright 0=0$.

2. $a \triangleright 1=\neg\ulcorner$.

3. $\ulcorner b\ulcorner a \Leftrightarrow a \triangleright 1 \leq b \triangleright 1$.

4. $a \triangleright p \leq p$.

5. $a \triangleright(a \triangleright p)=a \triangleright p$.

6. $(a+b) \triangleright p=(a \triangleright p) \cdot(b \triangleright p)$.

7. $b \leq a \Rightarrow a \triangleright p \leq b \triangleright p$, i.e., $\triangleright$ is antitone in its first argument.

8. $1 \leq a \Rightarrow a \triangleright p=0$.

So far, we have not required any special properties of the elements $a$ that represent, e.g., preference relations. Instead of d-transitivity or d-irreflexivity we need an assumption that such elements admit "enough" maximal objects. This is expressed by requiring every non-maximal object to be dominated by some maximal one. In a setting with finitely many objects, such as a database, and a preference relation on them this property is always satisfied. We will treat the case of infinite sets in Theorem 3.9 and Cor. 3.11 where we establish a connection between the notions of normality and being noetherian.

Definition 3.5 An element $a$ is called normal [7] if $\forall p:|a\rangle p \leq|a\rangle(a \triangleright p)$, meaning that every object dominated by some object of $p$ is also dominated by a maximal object of $p$. By $a \triangleright p \leq p$ and isotony of diamond this is equivalent to

$$
\forall p:|a\rangle p=|a\rangle(a \triangleright p) .
$$


One of the most important applications of normality is the following law.

Theorem 3.6 Let a be normal. Then $a \triangleright(p+q)=a \triangleright(a \triangleright p+a \triangleright q)$.

This theorem paves the way for a distributed computation of maxima, as for disjoint $p$ and $q$ the calculations $a \triangleright p$ and $a \triangleright q$ are independent. Early examples are found in [2, 10, further ones again in [1]. For a proof of the theorem see [7]; it generalises from + to arbitrary existing suprema in test $(S)$.

Next we present the announced connection between noetherity and the existence of maximal elements which will be used in Sect. 6.

Definition 3.7 An object $a$ is called noetherian if, for all tests $p$,

$$
a \triangleright p \leq 0 \Rightarrow p \leq 0
$$

This definition can be understood as follows. By contraposition and leastness of 0 it is equivalent to

$$
p \neq 0 \Rightarrow a \triangleright p \neq 0,
$$

which means that every non-empty $p$ contains at least one maximal object (which is the dual of the usual well-foundedness condition). In the relational case it is therefore also equivalent to the absence of infinitely ascending chains. For details see 4]. The following properties are straightforward by Boolean algebra.

Corollary 3.8 Assume an element a.

1. For arbitrary test $p$ we have $a \triangleright p \leq 0$ iff $p \leq|a\rangle p$.

2. $a$ is Noetherian iff for all tests $p$ we have $p \leq|a\rangle p \Rightarrow p \leq 0$.

Theorem 3.9 Let $a \in S$ be noetherian and let $a^{*}$ be its reflexive and transitive closure. Then for any $q \in \operatorname{test}(S)$ we have $q \leq\left|a^{*}\right\rangle(a \triangleright q)$. Informally, this means that any point in the set abstractly represented by $q$ is dominated w.r.t. $a^{*}$ by some point in $a \triangleright q$.

$$
\begin{aligned}
& \text { Proof. } \quad q \leq\left|a^{*}\right\rangle(a \triangleright q) \\
& \Leftrightarrow \quad\{\text { Boolean algebra }\} \\
& q-\left|a^{*}\right\rangle(a \triangleright q) \leq 0 \\
& \Leftarrow \quad\{\text { noetherity of } a \text { and Corollary } 3.82]\} \\
& q-\left|a^{*}\right\rangle(a \triangleright q) \leq|a\rangle\left(q-\left|a^{*}\right\rangle(a \triangleright q)\right) \\
& \Leftrightarrow \quad\{\text { Boolean algebra }\} \\
& q \leq\left|a^{*}\right\rangle(a \triangleright q)+|a\rangle\left(q-\left|a^{*}\right\rangle(a \triangleright q)\right) \\
& \Leftrightarrow \quad\{\text { star-dia-unfold and distributivity ]\} } \\
& q \leq a \triangleright q+|a\rangle\left|a^{*}\right\rangle(a \triangleright q)+|a\rangle\left(q-\left|a^{*}\right\rangle(a \triangleright q)\right) \\
& \Leftrightarrow \quad\{\text { Boolean algebra and distributivity }\} \\
& q-a \triangleright q \leq|a\rangle\left(\left|a^{*}\right\rangle(a \triangleright q)+\left(q-\left|a^{*}\right\rangle(a \triangleright q)\right)\right. \\
& \Leftrightarrow \quad\{\text { Boolean algebra }\} \\
& q \cdot|a\rangle q \leq|a\rangle\left(\left|a^{*}\right\rangle(a \triangleright q)+q\right)
\end{aligned}
$$




$$
\begin{array}{ll}
\Leftarrow & \{[\text { lattice algebra }\} \\
|a\rangle q \leq|a\rangle\left(\left|a^{*}\right\rangle(a \triangleright q)+q\right) \\
\Leftarrow \quad\{[\text { isotony of diamond }\} \\
& q \leq\left|a^{*}\right\rangle(a \triangleright q)+q \\
\Leftrightarrow & \{[\text { lattice algebra }\} \\
& \text { TRUE } .
\end{array}
$$

Corollary 3.10 If a is noetherian and d-transitive then for all tests $p, q$ we have

$$
\begin{gathered}
q \leq|a+1\rangle(a \triangleright q), \\
p \leq|a+1\rangle q \Leftrightarrow a \triangleright p \leq|a+1\rangle q .
\end{gathered}
$$

Proof. The first claim follows from Th. 3.9 and (2).

For the second claim, $(\Rightarrow)$ follows from $a \triangleright p \leq p$. For $(\Leftarrow)$ we have, by the first claim, the assumption with isotony of diamond and finally d-transitivity of $a$ and hence of $a+1$ that $p \leq|a+1\rangle(a \triangleright p) \leq|a+1\rangle|a+1\rangle q \leq|a+1\rangle q$.

This allows a much shorter proof of the following property from 7 .

Corollary 3.11 A noetherian and d-transitive element is normal.

Proof. For arbitrary test $q$ we obtain by Cor. 3.10 isotony of diamond and Cor. 3.2 that $q \leq|a+1\rangle(a \triangleright q) \Rightarrow|a\rangle q \leq|a\rangle|a+1\rangle(a \triangleright q) \Leftrightarrow|a\rangle q \leq|a\rangle(a \triangleright q)$.

In [7] it is also proved that every normal element is noetherian and dtransitive. We conclude with a further property of d-transitive elements.

Lemma 3.12 If $a$ is d-transitive then for all $p$ we have $a \triangleright(|a+1\rangle p)=a \triangleright p$.

$$
\begin{aligned}
& \text { Proof. } \quad a \triangleright(|a+1\rangle p) \\
& =\quad\{\text { definition of } a \triangleright]\} \\
& |a+1\rangle p-|a\rangle|a+1\rangle p \\
& =\{\text { Cor. } 3.2]\} \\
& (|a\rangle p+p)-|a\rangle p \\
& =\{[\text { right distributivity of }-]\} \\
& (|a\rangle p-|a\rangle p)+(p-|a\rangle p) \\
& =\quad\{\text { Boolean algebra }]\} \\
& p-|a\rangle p \\
& =\quad\{\text { definition of } a \triangleright]\} \\
& a \triangleright p \text {. }
\end{aligned}
$$

\section{Antichains}

An antichain is a set $M$ of objects of a partially ordered set such that any two objects of $M$ are incomparable. Equivalently, $M$ is an antichain if it coincides with its set of maximal elements, characterised algebraically as follows. 
Definition 4.1 Given a semiring object $a$, a test $p$ is an $a$-antichain if $p=a \triangleright p$, i.e., if $p$ is a fixed point of the operator $a \triangleright$. The set of all $a$-antichains is denoted by $\mathrm{AC}(a)$. By Lm. 3.4.1 $0 \in \mathrm{AC}(a)$ for every $a$. When $a$ is clear from the context we will just write "antichain" instead of " $a$-antichain".

Lemma $4.2 p$ is an antichain $\Leftrightarrow p \leq \neg|a\rangle p \Leftrightarrow p \cdot|a\rangle p \leq 0$. In particular, if a is $d$-irreflexive then every atomic test is an antichain.

Proof. By the definition of $\triangleright$, order theory, definition of,$- \cdot$ coinciding with infimum on tests, reflexivity of $\leq$ and Boolean algebra,

$$
\begin{aligned}
& p=a \triangleright p \Leftrightarrow p=p-|a\rangle p \Leftrightarrow p \leq p-|a\rangle p \Leftrightarrow \\
& p \leq p \wedge p \leq \neg|a\rangle p \Leftrightarrow p \leq \neg|a\rangle p \Leftrightarrow p \cdot|a\rangle p \leq 0 .
\end{aligned}
$$

Corollary 4.3 $\mathrm{AC}(a)$ is downward closed, i.e., $p \in \mathrm{AC}(a) \wedge q \leq p \Rightarrow q \in \mathrm{AC}(a)$.

Proof. By isotony we have $q \cdot|a\rangle q \leq p \cdot|a\rangle p \leq 0$.

Lemma 4.4 Consider tests $p, q$. Then $p+q$ is an antichain iff $p$ and $q$ are antichains and $p \cdot|a\rangle q \leq 0 \wedge q \cdot|a\rangle p \leq 0$.

Proof. $\quad p+q$ antichain

$$
\begin{array}{ll}
\Leftrightarrow & \{\text { by Lm. } 4.2]\} \\
& (p+q) \cdot|a\rangle(p+q) \leq 0 \\
\Leftrightarrow & \{[\text { distributivity }\} \\
& p \cdot|a\rangle p \leq 0 \wedge p \cdot|a\rangle q \leq 0 \wedge q \cdot|a\rangle p \leq 0 \wedge q \cdot|a\rangle q \leq 0 \\
\Leftrightarrow & \{\text { by Lm. } 4.2]\} \\
& p, q \text { antichains } \wedge p \cdot|a\rangle q \leq 0 \wedge q \cdot|a\rangle p \leq 0 .
\end{array}
$$

Lemma 4.5 For $p, q \in \mathrm{AC}(a)$ we have $a \triangleright(p+q)=(p-|a\rangle q)+(q-|a\rangle p)$.

$$
\begin{aligned}
& \text { Proof. } \quad a \triangleright(p+q) \\
& =\{\text { definition of } \triangleright\} \\
& (p+q)-|a\rangle(p+q) \\
& =\{\text { distributivity }\} \\
& (p+q)-(|a\rangle p+|a\rangle q) \\
& =\{[\text { De Morgan }\} \\
& (p+q)-|a\rangle p-|a\rangle q \\
& =\{\text { distributivity and right-commutativity of }-]\} \\
& (p-|a\rangle p-|a\rangle q)+(q-|a\rangle q-|a\rangle p) \\
& =\{[p, q \text { antichains and Lm. 4.2 }\} \\
& (p-|a\rangle q)+(q-|a\rangle p) \text {. }
\end{aligned}
$$




\section{Deriving the BNL Algorithm}

We now give an algebraic, calculational derivation of the BNL algorithm in [1] for computing the maximal objects of a set. For this, we assume that the test algebra of the underlying semiring is finite and hence atomic, i.e., every test is the sum of the atoms below it.

Consider a test $r$ that represents all available tuples in a database and let $a$ be a fixed strict-order representing a preference relation. The task is to compute $a \triangleright r$, i.e., a test representing the set of all $a$-maximal objects in $r$.

A common technique for deriving an algorithmic solution of a specification is to make a constant of the specification into a parameter and then calculate an inductive or recursive version of the generalised specification.

Here we make $r$ into a parameter called $u$. So for test $u$ we define the function $m a(u)$ that computes the maxima of $u$ w.r.t. preference $a$ as

$$
m a(u)=_{d f} a \triangleright u .
$$

The aim is now to develop a recursive version of the function $m a$ by induction on the size of the parameter $u$. By the assumptions of finiteness and atomicity the size $|u|$ of $u$ can be defined as the cardinality of the set of atoms below $u$.

Base Case $|u|=0$. Then $u=0$ and we have $m a(0)=0-|a\rangle 0=0$.

Inductive Case. Choose an atomic test $x \leq u$ and set $v=_{d f} u-x$. By the definitions, Th. 3.6 d-irreflexivity of $a$, atomicity of $x$, and the definition of $m a$ :

$$
m a(u)=a \triangleright(x+v)=a \triangleright(a \triangleright x+a \triangleright v)=a \triangleright(x+a \triangleright v)=a \triangleright(x+m a(v)) .
$$

Now we observe that $a \triangleright v$ is an antichain and define an auxiliary function

$$
\operatorname{inc}(x, p)=_{d f} a \triangleright(x+p),
$$

where $x$ is an atomic test and $p$ an antichain. Then we can continue the above derivation to obtain $m a(u)=\operatorname{inc}(x, m a(v))$.

Altogether, we have derived the recursion

$$
\begin{aligned}
m a(u)=\text { if } u=0 \text { then } 0 \\
\text { else choose atom } x \leq u \text { in } \\
\\
\quad \operatorname{inc}(x, m a(u-x)) .
\end{aligned}
$$

Our original task is now solved using the call $m a(r)$. We will transform this recursion into a simpler one in Sect. 6.

Next we derive a recursive version of the function $\operatorname{inc}(x, p)$. The parameter $p$ is frequently called the (working) window. It contains candidates for objects of the overall maxima set and is incrementally adapted as the single tuples $x$ are inspected in turn.

Base Case $|p|=0$ and hence $p=0$ : we have $\operatorname{inc}(x, 0)=a \triangleright(x+0)=a \triangleright x=x$.

Inductive Case: choose an atomic test $y \leq p$ and set $q=_{d f} p-y$. 
Subcase 1: $x \leq|a\rangle y$, i.e., $x$ is dominated by $y$. Therefore $x$ cannot be maximal in $r$ and can be discarded. Let us show this formally. First, by isotony of diamond, $x \leq|a\rangle p$, since $y \leq p$, and hence $x-|a\rangle p \leq 0$ by Boolean algebra. Moreover, again by isotony of diamond, d-transitivity of $a$ and $p$ being an antichain,

$$
p \cdot|a\rangle x \leq p \cdot|a\rangle|a\rangle p \leq p \cdot|a\rangle p \leq 0 .
$$

By Boolean algebra therefore $p \leq \neg|a\rangle x$ and hence $p-|a\rangle x=p$. Now Lm. 4.5 with $q$ specialised to $x$ shows $\operatorname{inc}(x, p)=p$.

Subcase 2: $x \not \leq|a\rangle y$. Then $x$ is not dominated by $y$ and cannot be discarded immediately. Rather, two subcases arise. If $x$ dominates $y$ then $y$ can be discarded from the window $p$. Otherwise $y$ still remains a candidate for a maximal object, while $x$ needs to be compared with the remainder $q$ of the window $p$. Again, we do the formal calculations.

First, since $x$ is an atomic test, (1) implies $x \leq \neg|a\rangle y$ and $x-|a\rangle y=x$.

Subcase 2.1: $y \leq|a\rangle x$ and hence $y-|a\rangle x=0$. By Lm. 4.5 distributivity, Boolean algebra and $\mathrm{Lm}$. 4.5 again we obtain

$$
\begin{aligned}
\operatorname{inc}(x, p) & =a \triangleright(x+y+q)=(x-|a\rangle(y+q))+((y+q)-|a\rangle x) \\
& =(x-|a\rangle y-|a\rangle q)+(y-|a\rangle x)+(q-|a\rangle x) \\
& =(x-|a\rangle q)+(q-|a\rangle x)=\operatorname{inc}(x, q) .
\end{aligned}
$$

Subcase 2.2: $y \not \leq|a\rangle x$, hence $y \leq \neg|a\rangle x$ and therefore $y \cdot|a\rangle x \leq 0$ and $y-|a\rangle x=$ $y$ by atomicity of $y$. Since $y \in p$ and $p$ is an antichain, we know that also $y \cdot|a\rangle q \leq 0$, hence $y \cdot|a\rangle(x+q) \leq 0$. Moreover, since we are in a case where $x \cdot|a\rangle y=0$, we know that also $(x+q) \cdot|a\rangle y \leq 0$. Now

$$
\begin{aligned}
& \text { inc }(x, p) \\
= & \{\text { by Lm. 4.5 }\} \\
& (x-|a\rangle p)+(p-|a\rangle x) \\
= & \quad\{\text { above decomposition } p=y+q, \text { additivity of diamond } \\
& \quad \text { and Boolean algebra }\} \\
& (x-|a\rangle y-|a\rangle q)+(y-|a\rangle x)+(q-|a\rangle x) \\
= & \{[\text { by } x-|a\rangle y=x \text { and } y-|a\rangle x=y, \text { as remarked above }]\} \\
= & (x-|a\rangle q)+y+(q-|a\rangle x) \\
& \quad\{\text { rearrangement and Lm. } \quad \text { is an antichain and } x \cdot q \leq x \cdot p \leq 0]\} \\
& y+i n c(x, q) .
\end{aligned}
$$

With this, the recursive version of inc is complete:

$$
\begin{aligned}
& \text { inc }(x, p)=\text { if } p=0 \text { then } x \\
& \text { else choose atom } y \leq p \text { in } \\
& \qquad \begin{array}{r}
\text { if } x \leq|a\rangle y \text { then } p \\
\text { else if } y \leq|a\rangle x \text { then } \operatorname{inc}(x, p-y) \\
\text { else } y+\operatorname{inc}(x, p-y) .
\end{array}
\end{aligned}
$$


We show the algorithm at work in our example from Fig. 1 in Sect. 1. The test $r$ represents the set of hotels (with abbreviated names) $\{\mathrm{GW}, \mathrm{WH}, \mathrm{OH}, \mathrm{A}, \mathrm{WW}, \mathrm{SSp}$, $\mathrm{RC}, \mathrm{H}, \mathrm{SH}\}$. The strict-order $a$ is the relation $\prec$. We show the values of $p, x$ and $y$, all in set notation, during the evaluation of the recursion for inc $(x, p)$ with initial values $x=\{\mathrm{SSp}\}$ and $p=\{\mathrm{GW}, \mathrm{H}, \mathrm{WH}, \mathrm{WW}\}$.

\begin{tabular}{|l||c|c|c|c|c|}
\hline step & 1 & 2 & 3 & 4 & 5 \\
\hline \hline$p$ & $\{\mathrm{GW}, \mathrm{H}, \mathrm{WH}, \mathrm{WW}\}$ & $\{\mathrm{GW}, \mathrm{H}, \mathrm{WH}\}$ & $\{\mathrm{H}, \mathrm{WH}\}$ & $\{\mathrm{WH}\}$ & $\emptyset$ \\
\hline$y$ & $\{\mathrm{WW}\}$ & $\{\mathrm{GW}\}$ & $\{\mathrm{H}\}$ & $\{\mathrm{WH}\}$ & - \\
\hline partial result & $\{\mathrm{WW}\}$ & $\{\mathrm{GW}\}$ & $\emptyset$ & $\{\mathrm{WH}\}$ & $x$ \\
\hline
\end{tabular}

In the first step we choose $y=\{\mathrm{WW}\}$. Then $x \nsubseteq|\prec\rangle y$ and $y \nsubseteq|\prec\rangle x$. Therefore $y$ is preserved as a partial result and the recursion continues with the remainder of the window. The second and fourth steps are analogous. In step $3 y$ is dominated by $x$ and hence discarded. Altogether, $\{\mathrm{GW}, \mathrm{SSp}, \mathrm{WH}, \mathrm{WW}\}$ is returned.

\section{$6 \quad$ The Lattice Structure of Antichains}

In this section we will exhibit a lattice structure on the set of antichains w.r.t. a strict-order. To this end we first define an approximation relation.

Definition 6.1 Test $p$ is improved by test $q$, in symbols $p \sqsubseteq q$, if $q$ results from removing some objects of $p$ that are dominated by $q$-objects and possibly adding others that are not dominated by $p$-objects. Formally,

$$
p \sqsubseteq q \Leftrightarrow_{d f} p-|a\rangle q \leq q \wedge q \cdot|a\rangle p \leq 0 .
$$

By Boolean algebra and distributivity we equivalently have

$$
p \sqsubseteq q \Leftrightarrow p \leq|a+1\rangle q \wedge q \cdot|a\rangle p \leq 0 .
$$

\section{Lemma 6.2}

1. $\forall p \in \operatorname{test}(S): 0 \sqsubseteq p$.

2. $\sqsubseteq$ is reflexive precisely on $\mathrm{AC}(a)$, i.e., $p \sqsubseteq p \Leftrightarrow p \in \mathrm{AC}(a)$.

3. $\sqsubseteq$ is antisymmetric.

4. If $a$ is d-transitive, then for antichains the second conjunct in the definition of $\sqsubseteq$ is implied by the first one, i.e., for $p, q \in \mathrm{AC}(a), p \sqsubseteq q \Leftrightarrow p \leq|a+1\rangle q$.

5. If $a$ is d-transitive then $\sqsubseteq$ is transitive and hence a partial order on $\mathrm{AC}(a)$.

6. If $a$ is normal then $p \sqsubseteq a \triangleright p$.

\section{Proof.}

1. Immediate from the definition and Lm. 4.2,

2 .

$$
\begin{aligned}
& p \sqsubseteq q \wedge q \sqsubseteq p \\
\Leftrightarrow & \quad\{\text { definition }\} \\
& p-|a\rangle q \leq q \wedge q \cdot|a\rangle p \leq 0 \wedge q-|a\rangle p \leq p \wedge p \cdot|a\rangle q \leq 0 \\
\Leftrightarrow \quad & \quad\{\text { commutativity of } \wedge \text { and Boolean algebra }\}
\end{aligned}
$$




$$
\begin{aligned}
& p-|a\rangle q \leq q \wedge p \leq \neg|a\rangle q \wedge q-|a\rangle p \leq p \wedge q \leq \neg|a\rangle p \\
\Leftrightarrow \quad & \{\mid \text { since } p \leq \neg|a\rangle q \wedge q \leq \neg|a\rangle p \text { imply } \\
& \quad p-|a\rangle q=p \text { and } q-|a\rangle p=q]\} \\
& p \leq q \wedge q \leq p \\
\Rightarrow \quad & \{\text { antisymmetry of } \leq \mathbb{\}} \\
& p=q .
\end{aligned}
$$

3. Assume $p \leq|a+1\rangle q$, which is equivalent to $p \leq|a\rangle q+q$. Then by isotony and distributivity of diamond, d-transitivity of $a$ and Lm. 4.2 ,

$$
q \cdot|a\rangle p \leq q \cdot(|a\rangle|a\rangle q+|a\rangle q)=q \cdot|a\rangle q=0 .
$$

4. By Part 4 isotony, d-transitivity of $a$ and hence of $a+1$, distributivity, and Part 4 again:

$$
\begin{aligned}
& p \sqsubseteq q \wedge q \sqsubseteq s \Leftrightarrow p \leq|a+1\rangle q \wedge q \leq|a+1\rangle s \\
& \quad \Rightarrow p \leq|a+1\rangle|a+1\rangle s \Rightarrow p \leq|a+1\rangle s \Leftrightarrow p \sqsubseteq s .
\end{aligned}
$$

5. By definition of $\sqsubseteq$, normality of $a(3)$, definition of $a \triangleright$ and Boolean algebra,

$$
\begin{gathered}
p \sqsubseteq a \triangleright p \Leftrightarrow p-|a\rangle(a \triangleright p) \leq a \triangleright p \wedge(a \triangleright p) \cdot|a\rangle p \leq 0 \Leftrightarrow p-|a\rangle p \leq \\
a \triangleright p \wedge(a \triangleright p) \cdot|a\rangle p \leq 0 \Leftrightarrow \operatorname{TRUE} .
\end{gathered}
$$

We show now that the order $\sqsubseteq$ holds, in particular, between $p$ and $i n c(x, p)$. Therefore the BNL algorithm produces a $\sqsubseteq$-ascending chain of antichains. It ends with the $\sqsubseteq$-largest antichain $a \triangleright r$, where $r$ is again the set of all tuples under consideration.

Theorem 6.3 Assume a to be a noetherian strict-order.

1. The operator a $\triangleright$ transforms all $\leq$-suprema existing in $\operatorname{test}(S)$ into $\sqsubseteq$-suprema in $\mathrm{AC}(a)$.

2. The operator $a \triangleright$ is isotone w.r.t. $\leq$ and $\sqsubseteq$, i.e.,

$$
\forall p, q \in \operatorname{test}(S): p \leq q \Rightarrow a \triangleright p \sqsubseteq a \triangleright q .
$$

3. $\mathrm{AC}(a)$ is an upper semilattice with $p \sqcup q=a \triangleright(p+q)$ and hence inc $(x, p)=p \sqcup x$ and $0 \sqcup p=p$.

4. If $(S, \leq)$ is a quantale then $\mathrm{AC}(a)$ is a complete lattice with $\bigsqcup_{\sqsubseteq} A=a \triangleright(\Sigma A)$, where $\Sigma$ is the supremum operator on $(S, \leq)$.

5. For atomic test $x$ with $x \cdot p=0$ and $p \in \operatorname{AC}(a)$ we have $p \sqsubseteq \operatorname{inc}(x, p)$.

6. The operator a $\triangleright$ preserves $\sqcup$ on $\operatorname{AC}(a)$.

7. The operator $a \triangleright$ is also isotone w.r.t. $\sqsubseteq$ and $\sqsubseteq$ on arbitrary tests, i.e.,

$$
\forall p, q \in \operatorname{test}(S): p \sqsubseteq q \Rightarrow a \triangleright p \sqsubseteq a \triangleright q .
$$

Proof. We recall the following characterisation of the supremum $s$ of a subset $X$ of a partially ordered set $M$ (provided it exists):

$$
\forall y \in M: s \leq y \Leftrightarrow(\forall x \in X: x \leq y) .
$$


1. Let $T \subseteq \operatorname{test}(S)$ have $\leq$-supremum $z$. Then

$$
\begin{aligned}
& \forall q \in a \triangleright T: q \sqsubseteq y \\
& \Leftrightarrow \quad\{\text { definition of } \sqsubseteq]\} \\
& \forall q \in a \triangleright T: q \leq|a+1\rangle y \\
& \Leftrightarrow \quad\{\text { definition of } a \triangleright T\} \\
& \forall p \in T: a \triangleright p \leq|a+1\rangle y \\
& \Leftrightarrow \quad\{\text { by Cor. } 3.10\} \\
& \forall p \in T: p \leq|a+1\rangle y \\
& \Leftrightarrow \quad\{\text { definition of } z\} \\
& z \leq|a+1\rangle y \\
& \Leftrightarrow \quad\{\text { by Cor. } 3.10\} \\
& a \triangleright z \leq|a+1\rangle y \\
& \Leftrightarrow \quad\{\text { definition of } \sqsubseteq\} \\
& a \triangleright z \sqsubseteq y .
\end{aligned}
$$

Hence, by (*), $a \triangleright z$ is the $\sqsubseteq$-supremum of the image set $a \triangleright T$ of $T$ under $a \triangleright$.

2. Immediate from Part 1 .

3. Immediate from Part 1 .

4. Immediate from Part 1 .

5. By d-irreflexivity of $a$ we have $x \in \mathrm{AC}(a)$. Hence Part 3 entails $p \sqsubseteq x \sqcup p=$ $a \triangleright(x+p)=\operatorname{inc}(x, p)$.

6. For $p, q \in \mathrm{AC}(a)$, by Part 3 idempotence of $\triangleright(\mathrm{Lm}$. 3.4.5), Th. 3.6, Part 3 .

$$
a \triangleright(p \sqcup q)=a \triangleright(a \triangleright(p+q))=a \triangleright(p+q)=a \triangleright(a \triangleright p+a \triangleright q)=a \triangleright p \sqcup a \triangleright q .
$$

7. For $p, q \in \operatorname{test}(S)$, by definition of $\sqsubseteq$, Part 2 and Lm. 3.12

$$
p \sqsubseteq q \Leftrightarrow p \leq|a+1\rangle q \Rightarrow a \triangleright p \sqsubseteq a \triangleright(|a+1\rangle q) \Leftrightarrow a \triangleright p \sqsubseteq a \triangleright q .
$$

It should be noted that noetherity is essential for these results. As a counterexample to $\leq$-isotony of $a \triangleright$, consider the semiring of binary relations on the set $\mathbb{N}$ of natural numbers. Take $a$ to be the usual strict-order on $\mathbb{N}$ so that $a+1$ is the standard order on $\mathbb{N}$. Choose as $p$ and $q$ the tests encoding $\{0\}$ and $\mathbb{N}$. Then $p \leq q$, but $a \triangleright p=p \nsubseteq \emptyset=a \triangleright q$, since $p \not \leq \emptyset=|a+1\rangle \emptyset$.

We conclude with an application of the algebra for bringing the function $m a$ from Sect. 5 into tail-recursive form, as a preparation for transliterating it into loop form (see e.g. 9] for details of that). The essential observation is that $\sqcup$ as a supremum operator is associative and has the $\sqsubseteq$-least element 0 as its neutral element. We define an auxiliary function $\operatorname{mat}(p, u)=_{d f} \quad p \sqcup \operatorname{ma}(u)$ with an additional parameter $p$ that will accumulate the end result during the recursion. By neutrality of 0 we can solve the original task as $m a(u)=\operatorname{mat}(0, u)$. Now we calculate a recursive version of mat based on the one for $m a$. In the termination case $u=0$ we obtain $\operatorname{mat}(p, 0)=p \sqcup 0=p$. In the recursive case for $u \neq 0$ we get by the definitions, Th. 6.3.3. associativity of $\sqcup$ and the definitions again

$$
\begin{gathered}
\operatorname{mat}(p, u)=p \sqcup \operatorname{inc}(x, \operatorname{ma}(u-x))=p \sqcup(x \sqcup \operatorname{ma} a(u-x))= \\
(p \sqcup x) \sqcup \operatorname{ma}(u-x)=\operatorname{mat}(p \sqcup x, u-x),
\end{gathered}
$$

which is a tail-recursive call. 


\section{Maxima as a Closure Operator}

\subsection{Closure Operators}

We recall the definition of a closure operator.

Definition 7.1 A closure operator on a partially ordered set $(L, \leq)$ is a total function $f: L \rightarrow L$ with the following properties:

$$
\begin{aligned}
& -x \leq f(x) \\
& -x \leq y \Rightarrow f(x) \leq f(y) \quad \text { (isotony) } \\
& \text { - } f(f(x))=f(x) \quad \text { (idempotence) }
\end{aligned}
$$

Consider now a noetherian strict-order $a$. By Lm6.2.6. Thm. 6.3.7 and Lm. 3.4.5 $a \triangleright$ satisfies all three of these properties w.r.t. $\sqsubseteq$. Unfortunately, however, $\sqsubseteq$ is not even a preorder on test $(S)$, since by $\mathrm{Lm}$. 6.2.2 reflexivity holds exactly on $\mathrm{AC}(a)$. To remedy this, we define another comparison relation on test $(S)$.

Definition 7.2 For a given $a$ we set $p \preceq_{a} q \Leftrightarrow_{d f} a \triangleright p \sqsubseteq a \triangleright q$.

Lemma $7.3 \preceq$ is a preorder, but not a partial order. We have $p \preceq q \wedge q \preceq p \Leftrightarrow$ $a \triangleright p=a \triangleright q$. Finally, $p \leq q \Rightarrow p \preceq q$.

Proof. Reflexivity and transitivity are immediate from reflexivity and transitivity of $\sqsubseteq$. The second claim follows from the antisymmetry of $\sqsubseteq$; it also shows that in general $\prec_{a}$ is not antisymmetric. The final claim is immediate from Th. 6.3 .2 and the definitions.

With this definition we can now actually view $a \triangleright$ as a closure operator if we carry over that notion to the case of preorders.

Definition 7.4 Consider a preorder $(L, \leq)$ with induced equivalence relation $\sim$. An endofunction $H: L \rightarrow L$ on a is called weakly idempotent if $H(H(x)) \sim H(x)$ for all $x \in L$. We call $H$ a kernel operator if it is isotone, weakly idempotent and contractive; by the latter property we mean $H(x) \leq x$ for all $x \in A$. Symmetrically, we call $H$ a closure operator if it is isotone, weakly idempotent and extensive; by the latter property we mean $x \leq H(x)$ for all $x \in A$.

In each case, the image set $H(A)$ coincides with the sets of weak fixed points of $H$, i.e., with the set $\{x \in A \mid H(x) \sim x\}$. Now we have the following result.

Lemma 7.5 a is a closure operator w.r.t. $\preceq$.

Proof. Since we already know that $a \triangleright$ is idempotent, it suffices to show extensivity and isotony w.r.t. $\preceq$.

Extensivity: by the definition of $\preceq$, idempotence of $a \triangleright$ (Lm. 3.4.5) and reflexivity of $\sqsubseteq$ we have $p \preceq a \triangleright p \Leftrightarrow a \triangleright p \sqsubseteq a \triangleright(a \triangleright p) \Leftrightarrow a \triangleright p \sqsubseteq a \triangleright p \Leftrightarrow$ TRUE.

Isotony: by the definition of $\preceq$, idempotence of $a \triangleright$ (Lm. 3.4.5) and the definition of $\preceq$ again we obtain

$$
p \preceq q \Leftrightarrow a \triangleright p \sqsubseteq a \triangleright q \Leftrightarrow a \triangleright(a \triangleright p) \sqsubseteq a \triangleright(a \triangleright q) \Leftrightarrow a \triangleright p \preceq a \triangleright q .
$$




\subsection{A Galois Connection for the Maxima Operator}

Since we have established the maxima operator as a closure operator, we can use a well-known result concerning Galois connections, again adapted to the case of preorders rather than partial orders.

Definition 7.6 Consider two preorders $\left(A, \leq_{A}\right)$ and $\left(B, \leq_{B}\right)$ and total functions $F: A \rightarrow B$ and $G: B \rightarrow A$. Then the pair $(F, G)$ is called a Galois connection $(G C)$ between $A$ and $B$ iff

$$
\forall x \in A: \forall y \in B: F(x) \leq_{B} y \Leftrightarrow x \leq_{A} G(y) .
$$

Then $F$ is called the lower, $G$ the upper adjoint of the GC.

Details of the theory of Galois connections for the preorder case can be found in the Appendix. The following Lm. is well known (e.g. [5]) for the case of partial orders; we adapt it to preorders.

Lemma 7.7 Every closure operator $H: L \rightarrow L$ induces the following Galois connection between $L$ and $H(L)$ :

$$
H(x) \leq y \Leftrightarrow x \leq \iota(y),
$$

where $\iota$ is the embedding of $H(L)$ into $L$, i.e., $\iota(y)=y$ for $y \in H(L)$.

Proof. $(\Rightarrow)$ By extensivity of $H$ and the assumption, $x \leq f(x) \leq y=\iota(y)$.

$(\Leftarrow)$ First, by weak idempotence of $H$ we have $H(y) \sim y$ for all $y \in H(L)$. Now, by isotony of $H$ we obtain $x \leq \iota(y) \Rightarrow H(x) \leq H(\iota(y))=H(y) \sim y$.

Hence for $p \in \operatorname{test}(S)$ and $q \in \mathrm{AC}(a)$ we have the Galois connection

$$
a \triangleright p \preceq q \Leftrightarrow p \preceq \iota(q) .
$$

As a lower adjoint therefore the $a \triangleright$ operator preserves all existing $\preceq$-suprema (see Th. 9.7 in the Appendix). This nicely rounds off the small collection of preservation results in Th. 6.3.

\section{Conclusion}

We have presented an algebraic account of an approximation relation between antichains that induces a semilattice and renders the maxima operator isotone in several ways. Moreover, the maxima operator has been shown to be a closure operator in an associated preordered set and hence satisfies a Galois connection. We have shown the calculus at work in the non-trivial example of the BNL algorithm. Therefore we are convinced that the theory developed here will be useful for many further calculational derivations involving the maxima operator and antichains.

Acknowledgment I am grateful to Peter Höfner, Martin E. Müller, Patrick Roocks, Andreas Zelend and the anonymous referees for careful proofreading and helpful remarks, and to Roland Backhouse for asking the questions that led to Sects. 6 and 7 He also suggested significant simplifications to an earlier version of this paper, in particular the property in Lm. 6.2.4. 


\section{References}

[1] Börzsönyi, S., Kossmann, D., Stocker, K.: The skyline operator. In: Data Engineering, 2001. Proceedings. 17th International Conference on. pp. 421-430 (2001)

[2] Dehne, F.: $o\left(n^{1 / 2}\right)$ algorithms for the maximal elements and ECDF searching problem on a mesh-connected parallel computer. Inf. Process. Lett. 22(6), 303306 (1986)

[3] Desharnais, J., Möller, B., Struth, G.: Kleene algebra with domain. ACM Trans. Comput. Logic 7(4), 798-833 (Oct 2006)

[4] Desharnais, J., Möller, B., Struth, G.: Algebraic notions of termination. Logical Methods in Computer Science 7(1) (2011)

[5] Erne, M., Koslowski, J., Melton, A., Strecker, G.: A primer on Galois connections. In: Proc. 1991 Summer Conference on General Topology and Applications in Honor of Mary Ellen Rudin and Her Work. Annals of the New York Academy of Sciences, vol. 704, pp. 103-125. New York Academy of Sciences (1993)

[6] Kozen, D.: A completeness theorem for Kleene algebras and the algebra of regular events. Information and Computation 110(2), 366-390 (1994)

[7] Möller, B., Roocks, P.: An algebra of database preferences. Journal of Logical and Algebraic Methods in Programming 84, 456-481 (2015)

[8] Möller, B., Roocks, P., Endres, M.: An Algebraic Calculus of Database Preferences. In: Gibbons, J., Nogueira, P. (eds.) Mathematics of Program Construction, Lecture Notes in Computer Science, vol. 7342, pp. 241-262. Springer Berlin Heidelberg (2012)

[9] Partsch, H.: Specification and Transformation of Programs - A Formal Approach to Software Development. Texts and Monographs in Computer Science, Springer (1990)

[10] Stojmenovic, I., Miyakawa, M.: An optimal parallel algorithm for solving the maximal elements problem in the plane. Parallel Computing 7(2), 249-251 (1988)

\section{Appendix: Galois Connections Between Preorders}

We investigate in how far the standard properties of Galois connections between partial orders hold for general preorders as well. For a good summary of the standard case see e.g. [5].

\subsection{Definition and Basic Properties}

Consider two preorders $\left(A, \leq_{A}\right)$ and $\left(B, \leq_{B}\right)$ and total functions $F: A \rightarrow B$ and $G: B \rightarrow A$. Then the pair $(F, G)$ is called a Galois connection $(G C)$ between $A$ and $B$ iff

$$
\forall x \in A: \forall y \in B: F(x) \leq_{B} y \Leftrightarrow x \leq_{A} G(y) .
$$

Then $F$ is called the lower, $G$ the upper adjoint of the GC.

In the sequel we shall suppress the indices of the preorders involved in a Galois connection.

The functions in a GC are quasi-inverses of each other: 
Lemma 9.1 (Quasi-Inverses (QI)) Assume that $F: A \rightarrow B$ and $G: B \rightarrow$ $A$ form a $G C$ between $A$ and $B$. Then

$$
\forall x \in A: x \leq G(F(x)), \quad \forall y \in B: F(G(y)) \leq y .
$$

Proof. By the GC and reflexivity $x \leq G(F(x)) \Leftrightarrow F(x) \leq F(x) \Leftrightarrow$ TRUE.

From (QI) we obtain

Corollary 9.2 (Isotony) The adjoints of a GC are isotone.

Proof. By transitivity, since QI entails $z \leq G(F(z))$, and GC:

$$
x \leq z \Rightarrow x \leq G(F(z)) \Leftrightarrow F(x) \leq F(z) .
$$

On the other hand, isotony and (QI) imply that we have a GC:

Lemma 9.3 (O. Ore) $(F, G)$ form a $G C$ iff $F$ and $G$ are isotone and quasiinverses of each other.

Proof. We only need to show the if-part. By isotony of $G$, and (QI):

$$
F(x) \leq y \Rightarrow G(F(x)) \leq G(y) \Rightarrow x \leq G(y) .
$$

Symmetrically one shows $x \leq F(y) \Rightarrow F(x) \leq y$.

For the following results we lift the equivalence $\sim$ induced by the preorder $\leq$ pointwise to functions by setting

$$
F_{1} \sim F_{2} \Leftrightarrow_{d f} \forall x: F_{1}(x) \sim F_{2}(x) .
$$

Then the rule of indirect equivalence immediately entails the following uniqueness property.

Lemma 9.4 (Determination) Let $\left(F_{i}, G_{i}\right)(i=1,2)$ be $G C s$ between $A$ and $B$. Then each adjoint determines the other one uniquely up to $\sim$, i.e.,

$$
F_{1} \sim F_{2} \Leftrightarrow G_{1} \sim G_{2} .
$$

Now we deal with iterated application of the adjoints.

Corollary 9.5 $F \circ G \circ F \sim F \quad$ and $G \circ F \circ G \sim G$.

Proof. From (QI) we know $x \leq G(F(x))$. Isotony implies $F(x) \leq F(G(F(x)))$. On the other hand, (QI) gives us $F(G(F(x))) \leq F(x)$, so that the claim follows by definition of $\sim$.

The claim on orders is immediate from that.

Corollary 9.6 Under the assumptions of Cor. 9.5, $F \circ G$ and $G \circ F$ are weakly idempotent and hence a closure and a kernel operator, respectively. 


\subsection{Galois Connections and Extremal Elements}

Consider an arbitrary preorder $(M, \leq)$. The sets of lower and upper bounds of a subset $X \subseteq M$ are given by

$$
y \in \operatorname{Iwb} X \Leftrightarrow_{d f} \forall x \in X: x \leq y, \quad z \in \operatorname{upb} X \Leftrightarrow_{d f} \forall x \in X: z \leq x .
$$

A quick calculation shows that upb $X \supseteq Y \Leftrightarrow X \subseteq$ Iwb $Y$. So (upb, Iwb) is a GC between $(\mathcal{P}(M), \supseteq)$ and $(\mathcal{P}(M), \subseteq)$.

Based on these we can define the sets of least and greatest objects of $X$ as

$$
\text { Ist } X=_{d f} X \cap \operatorname{lwb} X, \quad \text { gst } X=_{d f} X \cap \text { upb } X .
$$

Finally, the sets of infima and suprema of $X$ are given as

$$
\inf X={ }_{d f} \operatorname{gst} \operatorname{lwb} X, \quad \sup X=_{d f} \text { Ist upb } X .
$$

Note that any of these sets may be empty. All objects in a set lst $X$ or gst $X$ are $\sim$-equivalent.

Theorem 9.7 Let $(F, G)$ form a $G C$. Then

1. $F$ preserves all existing suprema, i.e., $F(\sup X) \subseteq \sup F(X)$ for all $X \subseteq A$.

2. $G$ preserves all existing infima, i.e., $G(\inf Y) \subseteq \inf F(Y)$ for all $Y \subseteq B$.

Proof. We only show 1. First,

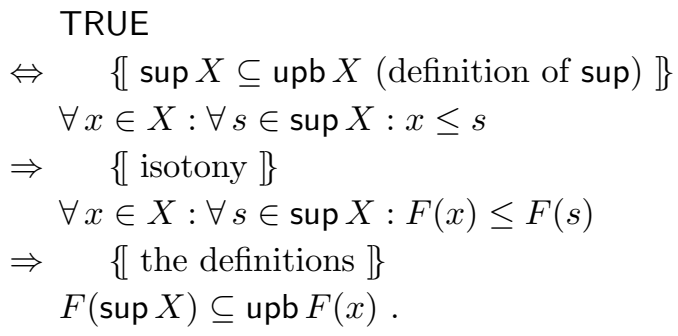

Second,

$$
\begin{aligned}
& \forall y \in \operatorname{upb} F(X): \forall x \in X: F(x) \leq y \\
& \Leftrightarrow \quad\{[\mathrm{GC}]\} \\
& \forall y \in \operatorname{upb} F(X): \forall x \in X: x \leq G(y) \\
& \Leftrightarrow \quad\{\text { definition }\} \\
& \forall y \in \operatorname{upb} F(X): G(y) \in \operatorname{upb} X \\
& \Leftrightarrow \quad\{\text { definition of sup }\} \\
& \forall y \in \operatorname{upb} F(X): \forall s \in \sup X: s \leq G(y) \\
& \Leftrightarrow \quad\{[\mathrm{GC}]\} \\
& \forall y \in \text { upb } F(X): \forall s \in \sup X: F(s) \leq y \\
& \Leftrightarrow \quad\{\text { definitions }\} \\
& F(\sup X) \subseteq \operatorname{Iwb} \operatorname{upb} F(X) \text {. }
\end{aligned}
$$

Now the claim is immediate from the definitions. 